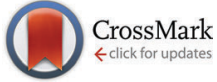

Cite this: Phys. Chem. Chem. Phys., 2015, 17, 12771

Received 28th February 2015 Accepted 9th April 2015

DOI: $10.1039 / c 5 c p 01213 a$

www.rsc.org/pccp

\section{Temperature-induced molecular transport through polymer multilayers coated with PNIPAM microgels $\uparrow$}

\author{
A. S. Vikulina, ${ }^{a b}$ S. T. Aleed, ${ }^{c}$ T. Paulraj, ${ }^{a}$ Yu. A. Vladimirov, ${ }^{b}$ C. Duschl, ${ }^{a}$ \\ R. von Klitzing ${ }^{\star C}$ and D. Volodkin*a
}

\begin{abstract}
Polyelectrolyte multilayers serve as effective reservoirs for bioactive molecules which are stored and released from the multilayers for cellular applications. However, control over the release without significantly affecting the multilayers and biomolecules is still a challenge. On the other hand, externally stimulated release would make the multilayers promising for the development of stimuli-sensitive planar carriers with release performance switched on demand. In this study soft composite films are designed by coating hyaluronic acid/poly-L-lysine (HA/PLL) multilayers with temperature responsive poly( $N$-isopropylacrylamide) (PNIPAM) microgels. Microgels are flattened and immersed into the multilayers to maximize the number of contacts with the surrounding polyelectrolytes (HA and PLL). The microgel coating serves as an efficient switchable barrier for the PLL transport into the multilayers. PLL diffusion into the film is significantly hindered at room temperature but is dramatically enhanced at $40{ }^{\circ} \mathrm{C}$ above the volume phase transition temperature (VPTT) of PNIPAM at $32{ }^{\circ} \mathrm{C}$ associated with microgel shrinkage. Scanning force microscopy micrographs show that the mechanism of volume phase transition on soft surfaces cannot be directly deduced from the processes taking place at solid substrates.
\end{abstract}

\section{Introduction}

The layer-by-layer polymer assembly ${ }^{1,2}$ is a very powerful and versatile tool for the fabrication of polymer-based multilayer films with tailor-made properties such as chemical composition, permeability, hydration, mechanics, etc. ${ }^{3-5}$ By the consecutive deposition of polycations and polyanions, the multilayer films can be assembled reaching film thicknesses from a few nanometers to several micrometers and beyond. Nowadays these multilayer films are extensively used as reservoirs to host bioactive molecules aiming at biological applications. ${ }^{6-10}$ A variety of molecules can be embedded into the films; they include small drugs and dyes, ${ }^{11-17}$ proteins and peptides, ${ }^{18-21}$ or nucleic acids. $^{22,23}$ Extensive reviews on the reservoir properties of the multilayers can be found elsewhere. ${ }^{6,7,10,24}$

Multilayers assembled from the biopolymers hyaluronic acid (HA) and poly-L-lysine (PLL) are some of the most studied

\footnotetext{
${ }^{a}$ Fraunhofer Institute for Cell Therapy and Immunology, Am Mühlenberg 13, 14476 Potsdam-Golm, Germany. E-mail: Dmitry.Volodkin@izi-bb.fraunhofer.de; Fax: +49-331-58187-399; Tel: +49-331-58187-327

${ }^{b}$ The Faculty of Fundamental Medicine, Laboratory of Medical Biophysics,

Lomonosov Moscow State University, Moscow, 119991, Russia

${ }^{c}$ Stranski-Laboratorium für Physikalische und Theoretische Chemie,

Technische Universität Berlin, Strasse des 17. Juni 124, D-10623 Berlin, Germany

$\dagger$ Electronic supplementary information (ESI) available. See DOI: 10.1039/c5cp01213a
}

systems. The HA/PLL multilayers are non-toxic for a variety of cell lines and have been employed to control cell adhesion via chemical or physical cues. ${ }^{11,25-29}$ The so-called exponential-like growth of the multilayers is caused by polymer diffusion in and out of the multilayers during the polymer assembly process. ${ }^{30-33}$ The exponential-like growth allows the production of micrometer thick films with high loading capacities for various biomolecules. $^{10,30}$ The multilayers can release biomolecules reaching the cells thereby inducing cellular response such as adhesion, migration or differentiation. .1,34,35 $^{-1}$

However, to stimulate a cellular response, it is necessary to ensure the delivery of biomolecules from the film to the cells. To date, stimuli-responsive multilayers have been prepared by employing polymers sensitive to $\mathrm{pH}$, ionic strength, and temperature variation. ${ }^{36,37}$ These stimuli, however, restrict the use of multilayers to biological experiments because even extreme environmental changes in $\mathrm{pH}$ and ionic strength can result in the improper functioning of cells or even cellular death. In addition, drastic changes in the film structure usually occur, which may impair the biological activity of the loaded biomolecules. Thus, control of the release performance is required without simultaneously changing the internal structure of the film. The concept of the present paper is based on embedding a stimuli responsive layer as a barrier, whose permeability can be switched without changing the structure of the surrounding multilayers. 
Stimuli-responsive materials play an important role in nonbiological and biological applications such as drug-delivery, sensing, diagnostics, tissue engineering, etc. ${ }^{38-43}$ Among others, thermo-responsive polymers are particularly attractive because they show a drastic change in physico-chemical characteristics as a result of phase transition upon heating. ${ }^{44,45}$ Poly( $N$-isopropylacrylamide) (PNIPAM) is one of the most well-known temperature sensitive polymers applied for the synthesis of macrogels and microgels (MG). ${ }^{46-49}$ At the volume phase transition temperature (VPTT at 32-34 ${ }^{\circ} \mathrm{C}$ ) PNIPAM chains undergo a reversible volume phase transition (VPT) mainly due to intramolecular hydrophobic interactions resulting in the release of bound water molecules and a pronounced volume reduction. ${ }^{50-54}$ The advantage of microgels vs. macrogels is that microgels react much faster to external stimuli (here: changes in temperature) than macrogels and they can be easily processed for film formation compared to macrogels. Temperature variations in the biocompatible range serve as a suitable stimulus because cells tolerate temperature shifts from 37 to $25^{\circ} \mathrm{C}^{43}$

In the present work, soft HA/PLL multilayer films are coated with a layer of negatively charged microgels. The polycation PLL is mobile within the HA/PLL film with a diffusion coefficient of up to $1 \mu \mathrm{m}^{2} \mathrm{~s}^{-1}$ and $\mathrm{HA}$ is rather immobile. ${ }^{19,55}$ The fast diffusion of PLL results in an excess of PLL at the surface of the HA/PLL film, leading to the strong interaction of HA/PLL films with negatively charged molecules. This phenomenon has been demonstrated for different species such as DNA, ${ }^{23,56}$ gold nanoparticles, ${ }^{57,58}$ liposomes ${ }^{59,60}$ as well as charged microparticles. $^{61,62}$ Macromolecules such as DNA are accumulated on the film surface forming large micrometer-sized aggregates with PLL. ${ }^{23,56}$ Negatively charged silica microparticles are spontaneously embedded into the HA/PLL films to about a half of their diameter. ${ }^{61}$ The silica particles are non-deformable and they can be embedded into the film just by immersing the film into particle suspension. The interaction of soft microgels with soft substrates has not been studied so far. In this study the interaction between HA/PLL multilayers and soft PNIPAM microgels (MG) is addressed. AFM is used to analyze the structure of the composite HA/PLL-MG films. For the first time we highlight PNIPAM microgel layers as barriers with temperature switchable permeability. Molecular transport through the composite film is examined by employing fluorescently labeled PLL.

\section{Experimental section}

\subsection{Synthesis of PNIPAM microgels}

The microgels were synthesized via precipitation polymerisation without using any surfactants. $N$-Isopropylacrylamide (NIPAM), $N, N^{\prime}$-methylenebisacrylamide (MBA), potassium persulfate (KPS) were obtained from Sigma-Aldrich. All chemicals were used without further purification. The purity of NIPAM was at least $97 \%$. The microgel particles were synthesized according to the procedure proposed in ref. 54. Both samples (MG1 and MG2) were synthesized with a 5 mol\% $N, N^{\prime}$-MBA crosslinker with respect to the molar amount of NIPAM (100 mM NIPAM and
$5 \mathrm{mM}$ MBA). Both reactions were initiated with $1 \mathrm{mM}$ of potassium persulfate (KPS). MG1 was prepared at a stirring rate of $500 \mathrm{rpm}$ with an immediate cold-purge at the end of the reaction followed by stirring at $500 \mathrm{rpm}$ overnight under nitrogen. MG2 was prepared during a reaction kinetic experiment, meaning that $2 \mathrm{~mL}$ sampling was performed at intervals throughout the reaction. The samples were slowly cooled down overnight under stirring at $1000 \mathrm{rpm}$. Both reactions were performed at a temperature of $80{ }^{\circ} \mathrm{C}$. Variation in the sample morphology was likely the result of reaction sampling, the method of terminating the reaction, and possibly also the stirring rate used.

\subsection{Characterization of PNIPAM microgels}

The hydrodynamic radii of MG1 and MG2 were measured by dynamic light scattering (DLS). The intensity auto-correlation functions were recorded at several scattering angles using an ALV/CGS-3 compact goniometer system equipped with an ALV/ LSE-5004 correlator and a He-Ne laser $(l=632.8 \mathrm{~nm}, 35 \mathrm{~mW})$. Data analysis was done using the inverse Laplace transformation algorithm (CONTIN). The zeta potential was measured using a Nano Zetasizer (Malvern).

\subsection{Preparation of $(\mathrm{HA} / \mathrm{PLL})_{24}$ films}

Soft HA/PLL multilayer films to be used as a host of microgels have been prepared according to a well-established protocol described in our previous studies. ${ }^{31,63,64}$ HA/PLL films were prepared on round $12 \mathrm{~mm}$ sized glass coverslips (Menzel, Germany). The coverslips were firstly cleaned in $2 \%$ Hellmanex solution followed by two times rinsing with $1 \mathrm{M} \mathrm{HCl}$ and subsequent two washing steps with water. Each cleaning step was done at $60{ }^{\circ} \mathrm{C}$ for $15 \mathrm{~min}$ incubation time. Cleaned slides were immersed in $1 \mathrm{mg} \mathrm{mL} \mathrm{mL}^{-1}$ polyethylenimine solution in $10 \mathrm{mM}$ TRIS containing $15 \mathrm{mM} \mathrm{NaCl}$ with $\mathrm{pH}$ adjusted to 7.4 (hereafter referred to as TRIS buffer) for $10 \mathrm{~min}$ followed by three times washing with buffer ( 3 min washing time). Furthermore, the coverslips were sequentially immersed in $0.5 \mathrm{mg} \mathrm{mL}^{-1}$ of HA and $0.5 \mathrm{mg} \mathrm{mL} \mathrm{mL}^{-1}$ of PLL solution in TRIS buffer for $10 \mathrm{~min}$. After every step of PLL or HA deposition the coverslips were washed three times with TRIS buffer for 3 min each time. The deposition cycles were repeated until the desired number of bilayers had been prepared. In this study (HA/PLL) ${ }_{24}$ films have been produced. The films were stored in TRIS buffer at $4{ }^{\circ} \mathrm{C}$. Before use the films have been "refreshed" by a sequence of PLL-HA-PLL deposition under the conditions used for the film assembly. The "refreshment" allows the minimization of the possible reorganization effects during film storage.

\subsection{Coating of (HA/PLL $)_{24}$ films with microgels}

(HA/PLL) ${ }_{24}$ films were exposed to $1 \mathrm{~mL}$ of $2 \mathrm{mg} \mathrm{mL}^{-1}$ microgel solution in TRIS buffer for $1 \mathrm{~h}$ followed by extensive washing with the buffer.

\subsection{AFM imaging of the coated multilayers}

AFM measurements in buffer were performed using the JPK NanoWizard ${ }^{\mathbb{R}}$ II in the intermittent contact mode. An electrochemical cell was used for measurements in buffer under 
temperature control. Mikromasch CSC37SPM cantilevers (C) were used for the measurements. The samples were stored at $4{ }^{\circ} \mathrm{C}$ before measurements were performed. AFM of both sample types was attempted at both 20 and $40{ }^{\circ} \mathrm{C}$. AFM image correction and analysis were conducted using Image software. The average values of microgel height and width have been obtained by the analysis of AFM image profiles obtained from a line profile or a 2D profile taken for at least 5 microgels, respectively (see Fig. 5 and Fig. S1, ESI $\dagger$ ).

\subsection{PLL diffusion into the (HA/PLL $)_{24}$ film}

A mixture of PLL-FITC with PLL (1:150 molar ratio) with a final polymer concentration of $0.5 \mathrm{mg} \mathrm{mL}^{-1}$ in TRIS buffer is added to the (HA/PLL) 24 -MG film deposited onto cover glass. The fluorescence increase in the film has been measured using a fluorescent microscope (Cell-R, Olympus). To examine the morphology of microgel coated films confocal scanning fluorescent microscopy (CLSM) has been employed (Zeiss LSM 510 Meta, Zeiss, Germany). The microscope is equipped with $63 \times$ (N.A. 1.4) oil-immersion objectives. Standard filter settings for excitation and emission of FITC were used for a laser source of $488 \mathrm{~nm}$.

\section{Results and discussion}

\subsection{Characterization of temperature-sensitive microgels}

Fig. 1 presents the hydrodynamic radius of the microgels as a function of temperature. At room temperature the hydrodynamic radii of MG1 and MG2 were found to be about $220 \mathrm{~nm}$, respectively. The microgels prepared by the procedure used in this study are negatively charged; the zeta-potential of MG2 was determined to be $-10 \mathrm{mV}$ and decreased to below $-35 \mathrm{mV}$ above VPTT (Fig. S2, ESI $\dagger$ ). The negative charge of the microgels is most probably induced by the polymerization initiator potassium persulfate (KPS) used to prepare the microgels. A volume phase transition at about $32{ }^{\circ} \mathrm{C}$ can be clearly identified by both size and zeta potential changes. The microgels shrink by almost a factor of two at temperatures above VPTT. This is a

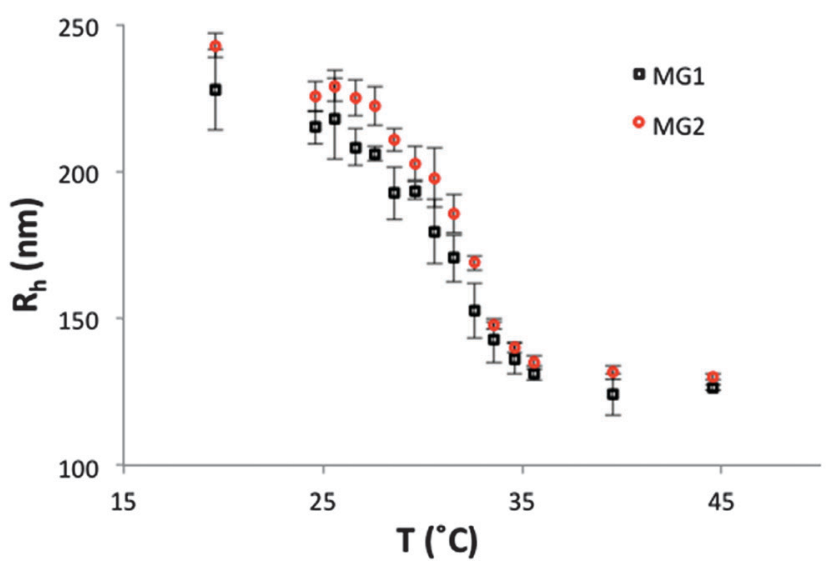

Fig. 1 Hydrodynamic radius of the microgels MG1 and MG2 as a function of temperature. typical behavior of microgels prepared by this approach as found in previous work (see Introduction). Shrunken microgels have higher negative zeta-potential values indicating a higher surface charge that is most probably caused by the reduction of surface area (Fig. 1). In addition, it is assumed that charges accumulate at the microgel surface during the volume phase transition. ${ }^{65}$

\subsection{Molecular transport through MG-coated HA/PLL films}

In the following both microgel samples MG1 and MG2 have further been studied as potential barrier materials for molecular transport into/out of the film. For this purpose PLL has been chosen as a model molecule, which can diffuse fast into the film and strongly accumulates in the film. The fluorescence of PLL diffused into the film has been probed by conventional fluorescence microscopy where the film thickness is much thinner than the field of depth. Therefore, the recorded fluorescent signal corresponds to the total fluorescence coming from the film and the fluorescence of a bulk above the film.

Fig. 2a shows the time evolution of fluorescence recorded from the film (coated with microgels or uncoated one) after its contact with PLL-FITC solution. The fluorescence is normalized by the signal obtained after the addition of PLL-FITC (burst).

A significant accumulation of PLL in the film is evident from Fig. $2 \mathrm{~b}$ due to much higher fluorescence from the film compared to the scratch area (where the film was removed). Three time segments can be distinguished in the fluorescence profiles in Fig. 2a. Interpretations of processes taking place in the segments are given in Fig. 3. Time segment I corresponds to a significant increase of fluorescence due to replacement of buffer solution above the film by PLL-FITC solution. This process is rather fast and lasts for less than $1 \mathrm{~min}$. Then, the fluorescence signal drops down. This corresponds to time segment II and can be explained by desorption of an excess of PLL-FITC molecules due to reorganization of the polymer structure at the film surface. The desorption of excess polymers has been previously reported using a quartz crystal microbalance allowing high temporal resolution measurements. ${ }^{66}$ The desorption process can last for a few minutes. On the MG-coated films no increase of fluorescence (MG1) or only a slight increase after $7 \mathrm{~min}$ (MG2) has been detected. In the case of the uncoated film, the fluorescence signal went up after approximately $3 \mathrm{~min}$ of incubation followed by a gradual slowing down with time. We attribute this to the polymer diffusion into the film (time segment III) as illustrated in Fig. 3. The increase of the fluorescence signal due to diffusion is significant because the film accumulates PLL.

Fig. 2a shows no fluorescence increase in the film with microgels adsorbed at the film surface; we explain this result by a barrier function of the microgel layer, which prevents PLL transport into the film (time segment III).

In order to investigate how the barrier characteristic of the microgel coating is changed at increased temperature, the PLL diffusion into the multilayers was studied at $40{ }^{\circ} \mathrm{C}$ (temperature above VPTT). Fig. 4 presents the evolution of fluorescence recorded from the coated and uncoated multilayers in the presence of PLL-FITC at $40{ }^{\circ} \mathrm{C}$. In contrast what happens at 

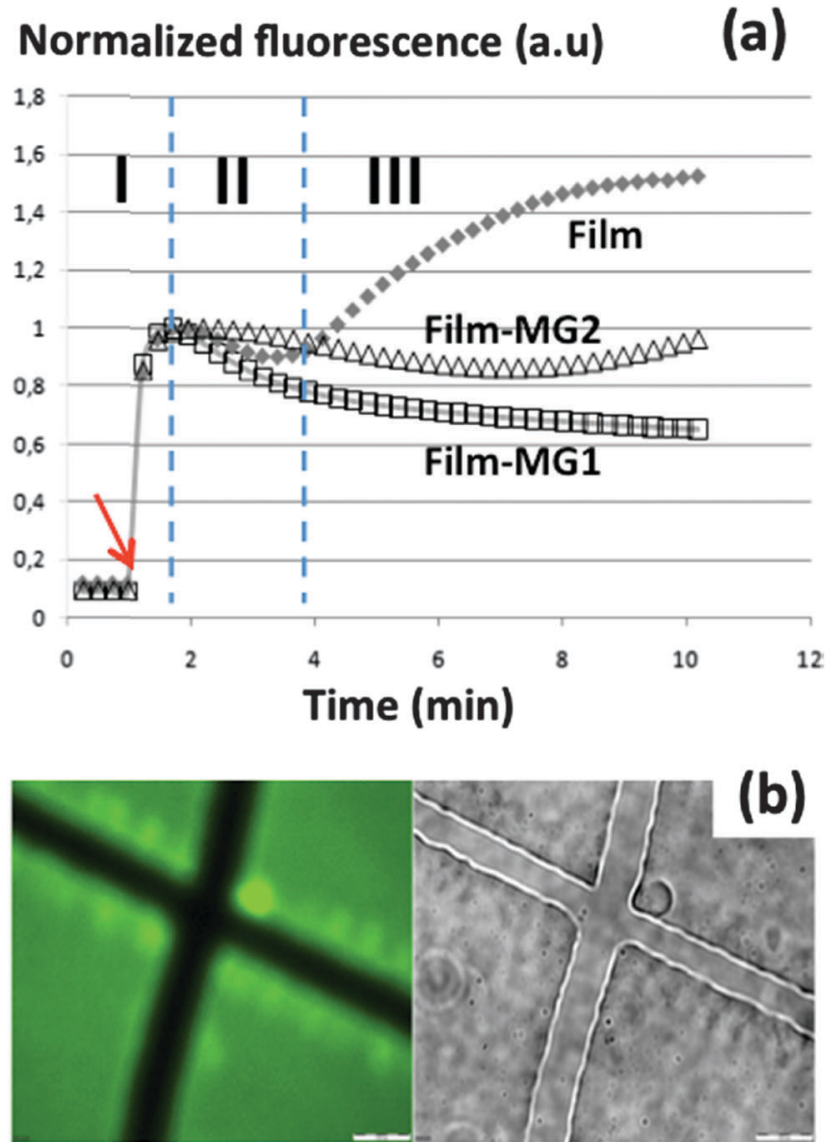

Fig. 2 Diffusion of PLL-FITC at $25^{\circ} \mathrm{C}$ : (a) normalized fluorescence detected from (HA/PLL) 24 films (uncoated or coated with MG1 and MG2) in contact with PLL-FITC. The processes associated with changes in the fluorescence signal are depicted: replacement of buffer with PLL solution as indicated by the red arrow (I), desorption of excess of adsorbed PLL (II), and PLL diffusion into the film (III). (b) CLSM (left) and transmission (right) images of a (HA/PLL) 24 film loaded with PLL-FITC are shown. The scratch made by a needle results in the mechanical removal of a part of the film and is used as a marker to identify the standard film position for fluorescence reading (the Z-position is changed until a maximum fluorescence signal is detected) and also as a control representing an uncoated surface. The scale bar for image (b) is $20 \mu \mathrm{m}$.

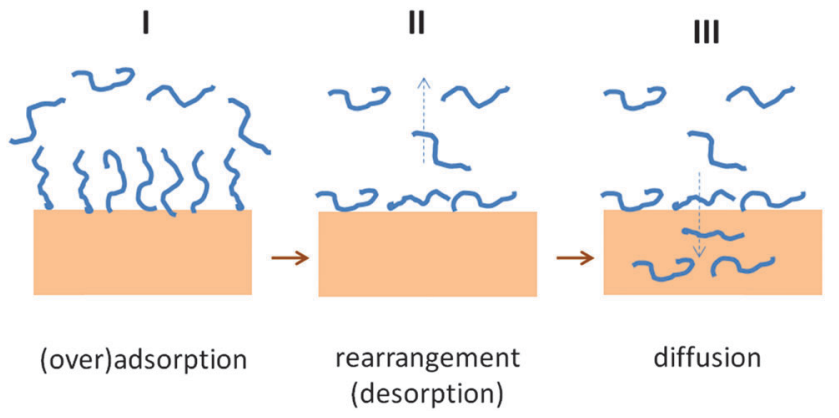

Fig. 3 Schematic presentation of polymer interaction with the film. The time segments I, II, and II represent polymer adsorption, desorption of an excess of polymer, and polymer diffusion into the film, respectively.

$25{ }^{\circ} \mathrm{C}$, now, the adsorption of microgels does not induce a significant change in the PLL transport into the multilayers. Interestingly, the shape of the fluorescence profile differs from

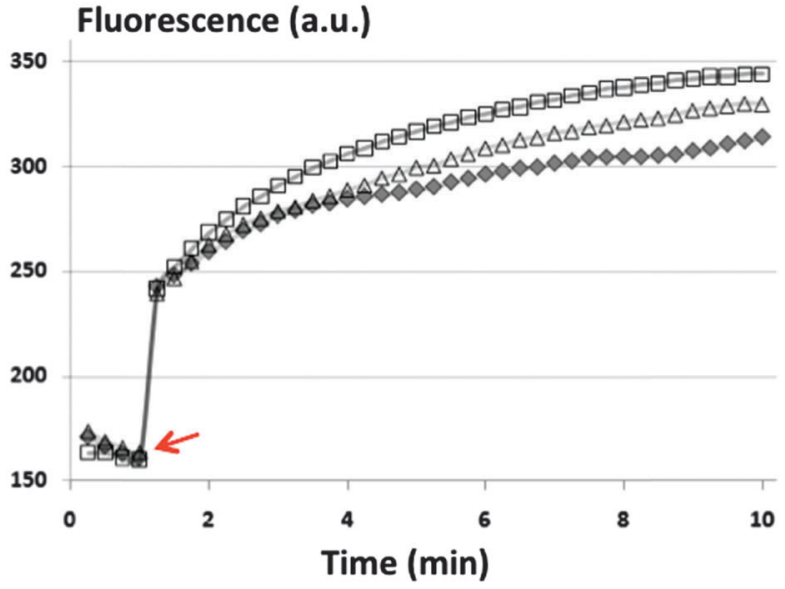

Fig. 4 Fluorescence detected from the uncoated (HA/PLL) 24 film (filled diamonds) and films coated with MG1 (open squares) and MG2 (open triangles) after contact with PLL-FITC at $40^{\circ} \mathrm{C}$. The red arrow shows the moment when PLL-FITC was added.

that measured at room temperature (Fig. 2a). Time segment II, where we assume desorption of the excess amount of adsorbed PLL (Fig. 3), is not observed at $40{ }^{\circ} \mathrm{C}$ (Fig. 4). This may be explained by a significant enhancement of PLL diffusion into the film (time segment III in Fig. 3). Thus, time segment II cannot be detected, since the signal due to PLL diffusion into the multilayers increases very fast from the very beginning.

Fig. 4 demonstrates the main finding of this study, namely that the microgels adsorbed at the multilayer surface serve as temperature-sensitive barriers. The barrier characteristics switch very much from negligible PLL transport below VPTT up to pronounced transport above the VPTT. Such "ON-OFF" behavior should be due to significant changes in the structure of the microgels. Another explanation for the stronger diffusion into the HA/PLL film might be that the microgels are (partially) desorbed after their volume phase transition. This is, however, not the case as shown in the next section.

\subsection{Volume phase transition of PNIPAM microgels adsorbed on HA/PLL multilayer films}

Fig. 5 and Fig. S1 (ESI $\dagger$ ) present AFM images of the surface of the microgel-coated HA/PLL films. The images have been recorded at 20 and $40{ }^{\circ} \mathrm{C}$ to probe the structure of the composite films below and above the VPTT of the microgels, respectively. White dots represent the top of the adsorbed microgels (Fig. 5). The most prominent result is that the microgels do not desorb from the HA/PLL film above the volume phase transition temperature. This means that the increased transport of PLL-FITC into the film above the VPTT in Fig. 4 cannot be explained by desorption of microgels but by a change in the volume of microgels. Therefore, the microgel structure was analysed more in detail in the AFM micrographs. The height and width of the MG1 and MG2 have been obtained from profile analysis as shown in Fig. 5b and are presented in Table 1.

Firstly, the height is much smaller than the width, indicating a flattening of the microgels at the surface. This is a common 

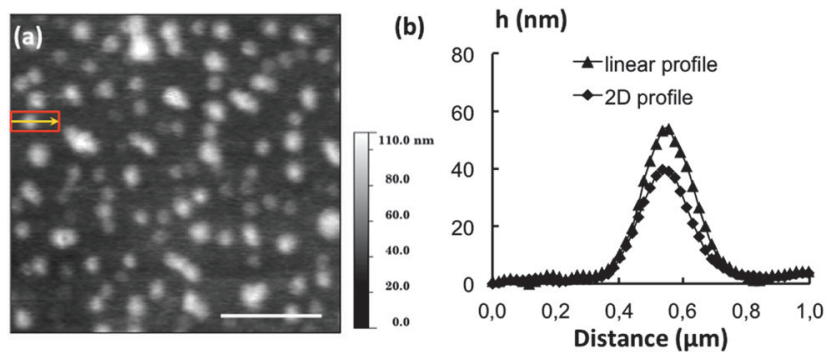

Fig. 5 AFM images of (HA/PLL) 24 films coated with MG2 (a) as measured at $20{ }^{\circ} \mathrm{C}$. (b) Linear and 2D profiles taken along the yellow line and a red rectangle in the image (a) to obtain microgel height and width, respectively.

Table 1 Height and width of the microgels MG1 and MG2 adsorbed on the $(\mathrm{HA} / \mathrm{PLL})_{24}$ multilayer film as measured by AFM at different temperatures $\left(20\right.$ and $\left.40{ }^{\circ} \mathrm{C}\right)$

\begin{tabular}{lll}
\hline Sample name & MG height $(h), \mathrm{nm}$ & MG width $(w), \mathrm{nm}$ \\
\hline MG1 $\left(20^{\circ} \mathrm{C}\right)$ & $24 \pm 5$ & $222 \pm 42$ \\
MG2 $\left(20^{\circ} \mathrm{C}\right)$ & $47 \pm 9$ & $314 \pm 26$ \\
MG1 $\left(40^{\circ} \mathrm{C}\right)$ & $15 \pm 6$ & $310 \pm 51$
\end{tabular}

behavior for all types of adsorbed PNIPAM microgels. On hard surfaces like silicon, glass or gold wafers a reduction in the volume by a factor of about $5-8$ is observed. ${ }^{46,67}$ This compression leads to a reduction in correlation length around the volume phase transition. ${ }^{68}$ In contrast to hard surfaces, the detected width of the microgel is reduced after adsorption (the diameter of microgels in solution is about $440 \mathrm{~nm}$ ). This is a hint that the microgel particles partially sink into the PEM, and that only the upper cap is imaged with the AFM tip. Upon increasing the temperature above the VPTT the detected width of MG1 increases (Table 1). This is in contrast to hard interfaces where a reduction in volume occurs, mainly due to vertical shrinking. The lateral dimension is often very similar to the diameter of the microgel in aqueous dispersion, but it depends on the interaction between the microgel and the hard surface. If there is any change of the lateral dimension at the VPT, it is always shrinking but the degree of shrinking strongly depends on the interaction between the microgel and the substrate (stick or slip) ${ }^{46,67}$ An increase in width when passing the VPT to higher temperatures has never been observed on hard substrates.

Obviously there is a mismatch between the detected increase in width at the VPT (Table 1) and the increase in mass transport, which indicates a lateral shrinking of the microgels. To explain the substantial enhancement of PLL transport above VPTT (Fig. 2 and 4) one can assume an increase in area devoid of microgels (area of voids). The PLL transport enhancement above the VPTT indicates a pronounced shrinking in the lateral direction. We considered the case of compactly packed spherical microgels and calculated an increase of surface available between the packed microgels when crossing VPTT if they would behave as in solution (Fig. S3, ESI $\dagger$ ). Most probably this behavior does not occur in our system due to interaction between microgels and the film. The spherical shape also does not correspond to the measured dimensions of the immobilized microgels. However, this simple model gives an upper limit for the maximum increase in the microgel-free area in the barrier microgel layer. Nevertheless, similar behavior is expected if the immobilized microgels are deformed (non-spherical). The calculation showed that in an ideal packing case the surface available between the microgels when heated above VPTT is enlarged by almost an order of magnitude (Fig. S3, ESI†). This can explain the dramatic enhancement of PLL transport into the film at temperature above VPTT.

As already mentioned above there are strong hints that the microgels partially sink into the HA/PLL film, and that only an upper cap of the microgel is detectable with the AFM tip. Due to the soft nature of the HA/PLL films, the microgels might be spontaneously immersed into the film. The increase in detected width above VPTT might be due to an increase in the elastic modulus of the microgel from about $100 \mathrm{kPa}$ to about $800 \mathrm{kPa},{ }^{46,69}$ which makes the detection of microgels easier by using the AFM. The value of Young's modulus of the surrounding HA/PLL film is in the range of tens of $\mathrm{kPa}^{64,70}$ The film structure is not changed upon heating to $45^{\circ} \mathrm{C}$ as has been proven from measurements of water content in the HA/PLL films upon heating. ${ }^{60}$ Thus, changes in the film permeability are related only to the change in the microgel structure.

The strong immersion into the PEM can be explained by the microgel structure. The microgels are assumed to have an inhomogeneous structure. Due to faster reaction kinetics of the cross-linker with respect to the NIPAM monomer, the microgel consists of a denser core surrounded by a more fluffy shell of dangling PNIPAM chains. ${ }^{71}$ Fig. 6 a schematically shows the structure of microgels adsorbed in the film. The dangling PNIPAM chains and the HA and PLL chains can strongly interdigitate with each other. Probably hydrogen bonds can be formed between the amide group of PNIPAM and the amide and carboxyl group of HA and the amine groups of PLL. Electrostatic attraction can also occur due to the negatively charged initiator of the PNIPAM microgel and the polycation PLL.

An annealing effect and a stronger interdigitation between the microgel and the surrounding HA/PLL film might occur at the temperature above VPTT. It is assumed that the immersed microgels keep the flattened shape and do not relax to a (a)

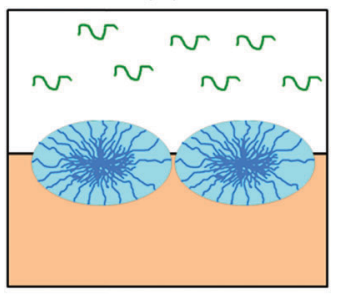

$20^{\circ} \mathrm{C}$

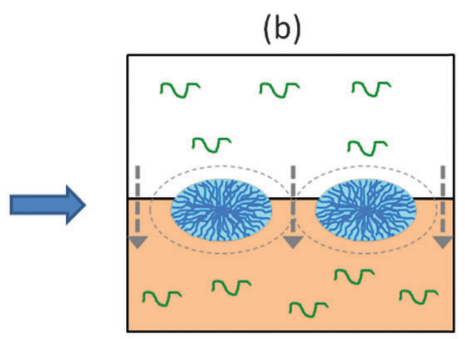

$40^{\circ} \mathrm{C}$
Fig. 6 Schematics (side view) of HA/PLL multilayers coated with PNIPAM microgels in the presence of PLL solution (green) at $20^{\circ} \mathrm{C}$ (a) and $40^{\circ} \mathrm{C}$ (b). The temperature increase above the phase transition results in the collapse of the immobilized microgels followed by an increase of the free surface area between the microgels. This facilitates the PLL diffusion into the multilayers (indicated by dashed arrows). 
spherical shape due to extra energy necessary to restructure the film. The film thickness (about $2 \mu \mathrm{m}^{14}$ ) is larger than the size of the microgels and even fully immersed microgels could not approach the solid support.

Fig. 6 represents schematics, which can explain temperature induced changes in the film-immobilized microgels, namely the measured widening of the microgels above VPPT. Microgel shrinkage upon heating from 20 to $40{ }^{\circ} \mathrm{C}$ undoubtedly occurs in the film, otherwise diffusion of PLL into the microgel coated and uncoated films could not be similar (Fig. 4). At $20{ }^{\circ} \mathrm{C}$ the dangling PNIPAM chains strongly interact with the surrounding polyelectrolytes (HA and PLL) in the film and the AFM can measure the size of just the denser (stiffer) core of the microgel. It results in a smaller detected size of the immobilized microgels compared to that of microgels in solution. The largest part of the microgel is immersed into the HA/PLL film and the measured microgel height is very low (Table 1). Above the VPPT the microgels shrink and allow PLL transport into the film (Fig. 6b). Now the dangling chains are retracted towards the shrinking gel core and contribute to the stiffer cap, which is detected by AFM.

In our future work we plan to further study the morphology of the composite films by using cantilevers with different spring constants. We would also like to consider reversibility of the temperature-mediated permeabilization and kinetics of microgel immersing into the film. This future study may shed light to the film structure.

\section{Conclusions}

This study shows that PNIPAM microgels can form an effective temperature sensitive barrier for molecular transport when adsorbed to HA/PLL multilayers. The negatively charged microgels are spontaneously adsorbed on the multilayer surfaces with being flattened and immersed with their largest part. Full immersion into the multilayers and relaxing to spherical shape would be unfavourable because of the necessary rearrangement of the internal structure of the multilayer. Dramatic enhancement of PLL transport at temperature above the VPTT of PNIPAM is due to the shrinking of the microgel particles at these temperatures. The composite soft films assembled from multilayers and PNIPAM microgels may serve as attractive models of composite structures for soft matter studies. This strategy offers new possibilities for the development of a novel generation of temperature-responsive materials for controlled drug release and the separation of compounds.

\section{Acknowledgements}

D.V. Volodkin thanks Alexander von Humboldt Foundation for support (Sofja Kovalevskaja Program). The work has been supported by DFG grants VO 1716/2-1 and KL 1165-12/1.

\section{References}

1 G. Decher, Science, 1997, 277, 1232-1237.

2 G. Decher and J.-D. Hong, Makromol. Chem., 1991, 46, 321.
3 Multilayer thin films: sequential assembly of nanocomposite materials, ed. J. B. S. Gero Decher, Wiley-VCH, 2nd edn, 2012.

4 R. von Klitzing, Phys. Chem. Chem. Phys., 2006, 8, 5012-5033.

5 R. Koehler, R. Steitz and R. von Klitzing, Adv. Colloid Interface Sci., 2014, 207, 325-331.

6 Z. Tang, Y. Wang, P. Podsiadlo and N. A. Kotov, Adv. Mater., 2006, 18, 3203-3224.

7 T. Boudou, T. Crouzier, K. Ren, G. Blin and C. Picart, Adv. Mater., 2010, 22, 441-467.

8 K. Ren, T. Crouzier, C. Roy and C. Picart, Adv. Funct. Mater., 2008, 18, 1-12.

9 V. Gribova, R. Auzely-Velty and C. Picart, Chem. Mater., 2011, 24, 854-869.

10 D. Volodkin, A. Skirtach and H. Mohwald, in Bioactive Surfaces, ed. H. G. Borner and J. F. Lutz, Springer-Verlag Berlin, Berlin, 2011, vol. 240, pp. 135-161.

11 C. Vodouhê, E. Le Guen, J. Mendez Garza, G. Francius, C. Déjugnat, J. Ogier, P. Schaaf, J.-C. Voegel and P. Lavalle, Biomaterials, 2006, 27, 4149-4156.

12 E. Nicol, J. L. Habib-Jiwan and A. M. Jonas, Langmuir, 2003, 19, 6178-6186.

13 S. E. Burke and C. J. Barrett, Macromolecules, 2004, 37, 5375-5384.

14 V. Z. Prokopović, C. Duschl and D. V. Volodkin, Macromol. Biosci., 2014, submitted.

15 V. Ball, Materials, 2012, 5, 2681-2704.

16 H. F. Chuang, R. C. Smith and P. T. Hammond, Biomacromolecules, 2008, 9, 1660-1668.

17 A. Schneider, C. Vodouhê, L. Richert, G. Francius, E. Le Guen, P. Schaaf, J.-C. Voegel, B. Frisch and C. Picart, Biomacromolecules, 2007, 8, 139-145.

18 X. F. Wang and J. Ji, Langmuir, 2009, 25, 11664-11671.

19 K. Uhlig, N. Madaboosi, S. Schmidt, M. S. Jager, J. Rose, C. Duschl and D. V. Volodkin, Soft Matter, 2012, 8, 11786-11789.

20 V. Kozlovskaya, E. Kharlampieva, I. Erel and S. A. Sukhishvili, Soft Matter, 2009, 5, 4077-4087.

21 M. L. Macdonald, R. E. Samuel, N. J. Shah, R. F. Padera, Y. M. Beben and P. T. Hammond, Biomaterials, 2011, 32, 1446-1453.

22 C. M. Jewell and D. M. Lynn, Adv. Drug Delivery Rev., 2008, 60, 979-999.

23 D. V. Volodkin, N. Madaboosi, J. Blacklock, A. G. Skirtach and H. Mohwald, Langmuir, 2009, 25, 14037-14043.

24 O. Guillame-Gentil, O. Semenov, A. S. Roca, T. Groth, R. Zahn, J. Voros and M. Zenobi-Wong, Adv. Mater., 2010, 22, 5443-5462.

25 O. V. Semenov, A. Malek, A. G. Bittermann, J. Voros and A. H. Zisch, Tissue Eng., Part A, 2009, 15, 2977-2990.

26 G. Francius, J. Hemmerlé, V. Ball, P. Lavalle, C. Picart, J.C. Voegel, P. Schaaf and B. Senger, J. Phys. Chem. C, 2007, 111, 8299-8306.

27 P. Podsiadlo, Z. Tang, B. S. Shim and N. A. Kotov, Nano Lett., 2007, 7, 1224-1231.

28 N. Madaboosi, K. Uhlig, S. Schmidt, M. S. Jager, H. Mohwald, C. Duschl and D. V. Volodkin, Lab Chip, 2012, 12, 1434-1436. 
29 C. Picart, Curr. Med. Chem., 2008, 17, 685-697.

30 P. Lavalle, J. C. Voegel, D. Vautier, B. Senger, P. Schaaf and V. Ball, Adv. Mater., 2011, 23, 1191-1221.

31 N. Madaboosi, K. Uhlig, M. S. Jäger, H. Möhwald, C. Duschl and D. V. Volodkin, Macromol. Rapid Commun., 2012, 33, 1775-1779.

32 C. Porcel, P. Lavalle, V. Ball, G. Decher, B. Senger, J.-C. Voegel and P. Schaaf, Langmuir, 2006, 22, 4376-4383.

33 D. Volodkin and R. von Klitzing, Curr. Opin. Colloid Interface Sci., 2014, 19, 25-31.

34 T. Crouzier, L. Fourel, T. Boudou, C. Albiges-Rizo and C. Picart, Adv. Mater., 2011, 23, H110.

35 T. Crouzier, A. Szarpak, T. Boudou, R. Auzely-Velty and C. Picart, Small, 2010, 6, 651-662.

36 S. Pavlukhina and S. Sukhishvili, Adv. Drug Delivery Rev., 2011, 63, 822-836.

37 S. A. Sukhishvili, Curr. Opin. Colloid Interface Sci., 2005, 10, 37-44.

38 H. Meng and G. Q. Li, J. Mater. Chem. A, 2013, 1, 7838-7865.

39 C. Fucinos, P. Fucinos, M. Miguez, I. Katime, L. M. Pastrana and M. L. Rua, PLoS One, 2014, 9, e87190.

40 J. L. Hu, H. P. Meng, G. Q. Li and S. I. Ibekwe, Smart Mater. Struct., 2012, 21, 053001.

41 B. R. Lee, H. J. Baik, N. M. Oh and E. S. Lee, Polymers, 2010, 2, 86-101.

42 M. A. Ward and T. K. Georgiou, Polymers, 2011, 3, 1215-1242.

43 E. Wischerhoff, K. Uhlig, A. Lankenau, H. G. Borner, A. Laschewsky, C. Duschl and J. F. Lutz, Angew. Chem., Int. Ed., 2008, 47, 5666-5668.

44 M. A. C. Stuart, W. T. S. Huck, J. Genzer, M. Muller, C. Ober, M. Stamm, G. B. Sukhorukov, I. Szleifer, V. V. Tsukruk, M. Urban, F. Winnik, S. Zauscher, I. Luzinov and S. Minko, Nat. Mater., 2010, 9, 101-113.

45 I. Tokarev, M. Motornov and S. Minko, J. Mater. Chem., 2009, 19, 6932-6948.

46 A. Burmistrova, M. Richter, M. Eisele, C. Uzum and R. von Klitzing, Polymers, 2011, 3, 1575-1590.

47 X. B. Zhang, C. L. Pint, M. H. Lee, B. E. Schubert, A. Jamshidi, K. Takei, H. Ko, A. Gillies, R. Bardhan, J. J. Urban, M. Wu, R. Fearing and A. Javey, Nano Lett., 2011, 11, 3239-3244.

48 T. L. Sun and G. Y. Qing, Adv. Mater., 2011, 23, H57-H77.

49 K. Kratz, T. Hellweg and W. Eimer, Polymer, 2001, 42, 6631-6639. 50 Y. L. Deng and R. Pelton, Macromolecules, 1995, 28, 4617-4621.

51 K. Kratz and W. Eimer, Ber. Bunsenges. Phys. Chem., 1998, 102, 848-854.
52 N. Dingenouts, C. Norhausen and M. Ballauff, Macromolecules, 1998, 31, 8912-8917.

53 H. Senff and W. Richtering, J. Chem. Phys., 1999, 111, 1705-1711.

54 R. Pelton, Adv. Colloid Interface Sci., 2000, 85, 1-33.

55 L. Jourdainne, S. Lecuyer, Y. Arntz, C. Picart, P. Schaaf, B. Senger, J. C. Voegel, P. Lavalle and T. Charitat, Langmuir, 2008, 24, 7842-7847.

56 D. Volodkin, A. Skirtach and H. Möhwald, Polym. Int., 2012, 61, 673-679.

57 D. V. Volodkin, M. Delcea, H. Mohwald and A. G. Skirtach, ACS Appl. Mater. Interfaces, 2009, 1, 1705-1710.

58 A. G. Skirtach, D. V. Volodkin and H. Mohwald, ChemPhysChem, 2010, 11, 822-829.

59 D. Volodkin, P. Schaaf, H. Mohwald, J.-C. Voegel and V. Ball, Soft Matter, 2009, 5, 1394-1405.

60 D. V. Volodkin, Y. Arntz, P. Schaaf, H. Mohwald, J.-C. Voegel and V. Ball, Soft Matter, 2008, 4, 122-130.

61 M. Delcea, N. Madaboosi, A. M. Yashchenok, P. Subedi, D. V. Volodkin, B. G. De Geest, H. Mohwald and A. G. Skirtach, Chem. Commun., 2011, 47, 2098-2100.

62 D. Kohler, N. Madaboosi, M. Delcea, S. Schmidt, B. G. De Geest, D. V. Volodkin, H. Möhwald and A. G. Skirtach, Adv. Mater., 2012, 24, 1095-1100.

63 V. Z. Prokopović, C. Duschl and D. V. Volodkin, Polym. Adv. Technol., 2014, 1342-1348.

64 C. Üzüm, J. Hellweg, N. Madaboosi, D. V. Volodkin and R. von Klitzing, Beilstein J. Nanotechnol., 2012, 3, 778-788.

65 S. Q. Zhou and B. Chu, J. Phys. Chem. B, 1998, 102, 1364-1371.

66 T. J. Lane, W. R. Fletcher, M. V. Gormally and M. S. Johal, Langmuir, 2008, 24, 10633-10636.

67 A. Burmistrova and R. von Klitzing, J. Mater. Chem., 2010, 20, 3502-3507.

68 S. Wellert, Y. Hertle, M. Richter, M. Medebach, D. Magerl, W. Wang, B. Deme, A. Radulescu, P. Muller-Buschbaum, T. Hellweg and R. von Klitzing, Langmuir, 2014, 30, 7168-7176.

69 A. Burmistrova, M. Richter, C. Uzum and R. von Klitzing, Colloid Polym. Sci., 2011, 289, 613-624.

70 S. Schmidt, N. Madaboosi, K. Uhlig, D. Köhler, A. Skirtach, C. Duschl, H. Möhwald and D. V. Volodkin, Langmuir, 2012, 28, 7249-7257.

71 E. Daly and B. R. Saunders, Phys. Chem. Chem. Phys., 2000, 2, 3187-3193. 